\title{
EUCLIDEAN NULL CONTROLLABILITY OF INFINITE NEUTRAL DIFFERENTIAL SYSTEMS
}

\author{
DAVIES IYAI ${ }^{1}$
}

(Received 1 May, 2006)

\begin{abstract}
This paper is aimed at establishing sufficient computable criteria for the Euclidean null controllability of an infinite neutral differential system, when the controls are essentially bounded measurable functions on finite intervals, with values in a compact subset $U$ of an $\boldsymbol{m}$-dimensional Euclidean space with zero in its interior. Our results are obtained by exploiting the stability of the free system and the rank criterion for properness of the controlled system. An example is also given.
\end{abstract}

2000 Mathematics subject classification: primary 93B05; secondary 34H05.

Keywords and phrases: controllability, neutral system, infinite delay, Euclidean null controllability.

\section{Introduction}

As seen in Davies and Jackreece [7] and the references therein, differential equations, are in general an important tool for harnessing different components into a single system and analysing the inter-relationships that exist between these different components which otherwise might continue to remain independent of each other. In fact, physical systems which express the present states of situations are the most common systems encountered in the theory of differential equations. However, a more realistic system should encompass not only the present but also the past states of the system. For a good grasp of the present $(t)$, some knowledge of the past $(t-r), t \geq 0$, is very important. Such systems were formulated by Volterra in 1928, when he took into account the past states of a system in his study of predator-prey models. This principle permeates various aspects of life and has of late influenced many researchers.

In general, differential equations which involve the present as well as the past state of any physical system are called delay differential equations or functional differential

\footnotetext{
${ }^{1}$ Department of Mathematics and Computer Science, Rivers State University of Science and Technology, P.M.B. 5080, Port Harcourt, Rivers State, Nigeria; e-mail: davsdone@ yahoo.com. (C) Australian Mathematical Society 2006, Serial-fee code 1446-1811/06
} 
equations. Delay differential equations can be classified into two broad types: retarded functional differential equations and neutral functional differential Equation (NFDE). Our interest is in the latter type, one in which the derivatives of the past history or derivatives of functionals of the past history are involved as well as the present states of the system. Several authors such as Xu et al. [15], Kuang and Feldstein [10] and Chukwu and Simpson [4] have studied the theory of neutral functional differential equations and have obtained independent results.

The controllability of neutral systems has been studied by several authors including Chukwu [3], Fu [8] and Gahl [9]. The control equations of linear neutral systems have applications in the study of electrical networks containing lossless transmission lines, electrodynamics, variational problems etc. (see Onwuatu [12]).

Owing to the difficulty that arises in presenting real-life situations in ecology, epidemics, population growth $e t c$., the study of integro-differential equations with infinite delay has emerged as a branch of modern research (see Burton [2], Corduneanu [5] and Lakshmikantham [11] for detail). These studies have been extended to the controllability of infinite neutral functional differential equations in recent years. In [12], Onwuatu studied a class of nonlinear infinite neutral system, where he developed sufficient conditions for the null controllability of such systems. Balachandran and Anandi [1] have studied the controllability of a class of neutral functional integrodifferential infinite delay systems in Banach spaces by using analytic semi-group theory and the Nussbaum fixed point theorem. Our objective in this research is to give sufficient conditions for Euclidean null controllability for infinite neutral differential systems with distributed delays of the form

$$
\left.\frac{d}{d t} D(t) x_{t}(\cdot)=L(t, x(\cdot), u(\cdot))+\int_{-\infty}^{0} A(\theta) x(t+\theta)\right) d \theta .
$$

Our results shall incorporate and extend other known results in the literature.

\section{Basic notation, preliminaries and definitions}

Suppose $h>0$ is a given number, $E=(-\infty, \infty), E^{n}$ is a real $n$-dimensional Euclidean space with norm $|\cdot|$ and $C=C\left([-h, 0], E^{n}\right)$ is the space of continuous function mapping the interval $[-h, 0]$ into $E^{n}$ with the norm $\|\cdot\|$, where $\|\phi\|=$ $\sup _{-h \leq s \leq 0}|\phi(s)|$, for $\phi \in C$. Let $\tau \in E, a>0$ and $x \in C\left([\tau-h, \tau+a], E^{n}\right)$. Then given $t \in[\tau, \tau+a]$, we define the symbol $x_{t}$ by $x_{t}(s)=x(t+s),-h \leq s \leq 0$. Let $g$ be a bounded linear operator taking $[\tau, \infty] \times C \rightarrow E^{n}$. We define the functional difference operator $D(\cdot):[\tau, \infty] \times C \rightarrow E^{n}$ by

$$
D(t) \phi=\phi(0)-g(t, \phi) .
$$


For $t \in[\tau, \infty], \phi \in C$, we now define a neutral functional differential equation to be a system of the form

$$
\frac{d}{d t} D(t) x_{t}=f\left(t, x_{t}\right)
$$

where $x_{t} \in C$, and $f$ is a continuous function from $(\tau, \infty) \times C$ into $E^{n}$. We say that $x$ is a solution of (2.2) with initial value $\phi$ at $\sigma$ if there exists $a \in[\tau, \infty], a>0$ such that $x \in C\left([\sigma-h, \sigma+a], E^{n}\right), x_{0}=\phi, D(t) x_{t}$ is continuously differentiable on $(\sigma, \sigma+a)$ and (2.2) is satisfied on $(\sigma, \sigma+a)$.

We shall consider control systems of the form

$$
\frac{d}{d t} D(t) x_{t}=L(t, x, u)+\int_{-\infty}^{0} A(\theta) x(t+\theta) d \theta
$$

The linear base control system is given by

$$
\frac{d}{d t} D(t) x_{t}=L(t, x, u)
$$

and the free system is given by

$$
\frac{d}{d t} D(t) x_{t}=L(t, x, 0)+\int_{-\infty}^{0} A(\theta) x(t+\theta) d \theta,
$$

where $D(t) x_{t}=x(t)-A x(t-1), L(t, x, \bar{u})=G x(t)+B x(t-1)+F u(t)+H u(t-h)$, $A, B, G$ are $n \times n$ matrices, $F, H$ are $n \times m$ matrices and $A(\theta)$ is an $n \times n$ matrix whose elements are square integrable on $(-\infty, 0]$.

Let $X(t)$ be the unique $n \times n$ constant matrix function with the following properties:

(a) $X(t)>0$, for $t<0$,

(b) $X(t)=I$, the identity matrix,

(c) $X(t)-A X(t-1)$ is continuous on $[0, \infty)$ and

(d) $X(t)$ satisfies $\dot{X}(t)-A \dot{x}(t-1)=L(x, 0)$,

for $t \in(0, \infty)-S_{2}$, where $S_{2}$ is the set of non-negative integers.

Then a unique solution of (2.4) exists on $[1, t]$ satisfying $x_{L}(t, u)=\phi(t)$ for $t \in[0,1]$ and by Gahl [9], this solution is given by

$$
\begin{aligned}
x_{L}(t, u)= & X(t-1) \phi(1)-X(t-2) \phi(1) \\
& +\int_{1}^{t} X(t-s-1)[A \phi(s)+B \phi(s)] d s \\
& +\int_{1}^{t} X(t-s)[F u(s)-H u(s-h)] d s
\end{aligned}
$$


for all $t \in\left[1, t_{1}\right]$, where $x_{L}(t, u)$ is a continuous function which satisfies (2.4) on $\left[1, t_{1}\right]$ except for a finite number of points which are contained in the set

$$
S_{3}=S_{2}\left\{t: t=t_{1} \pm h+I ; t \neq k \text { or } h \neq I, \text { for } k \in S_{2}\right\}
$$

Define the matrix functions $Z$ by

$$
Z(t, s)=X(t, s) F+X(t-s-h) H .
$$

Then it follows immediately that

$$
x_{L}(t, u)=x_{L}(t, 0)+\int_{1}^{t} Z(t, s) u(s) d s .
$$

Since $X(t)$ is continuous and bounded on $[a, b]-S_{2}$,

$$
\frac{\partial}{\partial t} Z(t, s)=X(t, s) F+X(t-s-h) H
$$

is continuous and bounded on $[a, b]-S_{2}$. In a similar manner, following the methods of Gahl [9] and Sinha [14], any solution of system (2.3) will be given by

or

$$
x(t, u)=x_{L}(t, u)+\int_{1}^{t} X(t, s) \int_{-\infty}^{0} A(\theta) x(t+\theta) d \theta d s
$$

$$
\begin{aligned}
x(t, u)= & x_{L}(t, 0)+\int_{1}^{t} Z(t, s) u(s) d s \\
& +\int_{1}^{t} X(t-s) \int_{-\infty}^{0} A(\theta) x(t+\theta) d \theta d s .
\end{aligned}
$$

In this paper, the control space will be $L_{\infty}^{\mathrm{loc}}\left([0, \infty), E^{m}\right)$, the space of essentially bounded measurable functions on finite intervals with values in $E^{m}$. The control constraint set will be denoted by $U=L_{\infty}^{\text {loc }}\left([0, \infty), C^{m}\right)$, where $C^{m}=\left\{u \in E^{m}\right.$ : $\left.\left|u_{j}\right| \leq 1, j=1, \ldots, m\right\}$

We now give some definitions upon which our study hinges.

DEFINITION 2.1. The solution $x=0$ of (2.5) is called stable at $t_{0}$ if $t_{0}>0$ and there exists a $b=b\left(t_{0}\right)$ such that if $\|\phi\| \leq b$, then the solution $x\left(t_{0}, \theta\right)$ exists for $t>t_{0}$ and $x_{t}\left(\cdot, t_{0}, \phi\right)$ is in the domain of definition $L(t, x, u)$ for $t \geq t_{0}$. Also, for each $\varepsilon>0$ there exists a $\delta=\delta\left(t_{0}, \phi\right)>0$ such that if $\|\phi\|<\delta$ then the solution $x\left(t_{0}, \theta\right)$ of (2.5) satisfies $\left\|x\left(t_{0}, \phi\right)\right\| \leq \varepsilon$ for $\phi \in C$. The trivial solution of (2.5) is called stable if it is stable for $t_{0} \geq 0$. It is called uniformly stable if it is stable and the $\delta$ above does not depend on $t_{0}$. It is uniformly asymptotically stable if it is uniformly stable and for every $\eta>0$ and every $t_{0}>0$ there exist $T(\eta)$ independent of $t_{0}$ and $H_{0}>0$ independent of $\eta, t_{0}$ such that $\|\phi\| \leq H_{0}$ implies $\left\|x\left(t_{0}, \phi\right)\right\| \leq \eta$, for all $t \geq t_{0}+T(\eta)$. 
DEFINITION 2.2. System (2.3) is said to be completely Euclidean controllable on $\left[t_{0}, t_{1}\right]$ if for each function $\phi \in C$, and each $x_{1} \in E^{n}$, there is an admissible control $u \in L_{\infty}\left(\left[t_{0}, t_{1}\right], E^{m}\right)$ such that the solution $x\left(t, t_{0}, u\right)$ of $(2.3)$ satisfies $x_{L_{0}}\left(\cdot, t_{0}, u\right)=\phi$, $x_{L}\left(t_{1}, t_{0}, u\right)=x_{1}$. It is Euclidean controllable on $\left[t_{0}, t_{1}\right]$ with constraints, if for each $x_{1} \in E^{n}, \phi \in C$, there exists a $t_{1}>t_{0}+h$ such that the solution of (2.3) satisfies $x_{L_{t_{0}}}\left(\cdot, t_{0}, u\right)=\phi, x_{L}\left(t_{1}, t_{0}, u\right)=x_{1}$.

DEFINITION 2.3. System (2.3) is Euclidean null controllable on $\left[t_{0}, t_{1}\right]$ if for each $\phi \in C$, there exists a $t_{1}>t_{0}$ and $u \in L_{\infty}\left(\left[t_{0}, t_{1}\right], E^{m}\right)$ such that the solution $x_{L}(t, u)$ of (2.3) satisfies $x_{L_{t_{0}}}(\cdot, u)=\phi$ and $x_{L}(t, u)=0 \in E^{n}, t_{1}>t_{0}$. It is Euclidean null controllable with constraints if, given any $\theta \in C$, there exist a $t_{1}>t_{0}$ and a $u \in L_{\infty}\left(\left[t_{0}, t_{1}\right], C^{m}\right)$ such that the solution $x_{L}(t, u)$ of $(2.3)$ satisfies $x_{L_{t_{0}}}(\cdot, u)=\phi$ and $x_{L}(t, u)=0$.

DEFINITION 2.4. The system (2.3) is proper on $\left[t_{0}, t_{1}\right]$ if $\eta^{T} Z(t, s)=0$ a.e. $s \in$ $\left[t_{0}, t_{1}\right]$ implies $\eta=0$ for $\eta \in E^{n}$, where $\eta^{T}$ is the transpose of $\eta$. If (2.3) is proper on each interval $\left[t_{0}, t_{1}\right]$, we say that the system is proper.

DEFINITION 2.5. The reachable set of (2.3) is a subset of $E^{n}$ given by

$$
P\left(t, t_{0}\right)=\left\{\int_{1}^{t} Z(t, s) u(s) d s: u \in L_{\infty}\left(\left[t_{0}, t_{1}\right], E^{m}\right)\right\} .
$$

If the controls are in $L_{\infty}\left(\left[t_{0}, t_{1}\right], C^{m}\right)$, we define the constraint reachable set by

$$
R\left(t, t_{0}\right)=\left\{\int_{1}^{t} Z(t, s) u(s) d s: u \in L_{\infty}\left(\left[t_{0}, t_{1}\right], C^{m}\right)\right\} .
$$

Note that $P\left(t, t_{0}\right)$ is a subset of $E^{n}$ which is symmetric about zero.

DEFINITION 2.6. The controllability matrix of (2.3) will be given by

$$
W=\int_{1}^{t} Z(t, s) Z^{T}(t, s) d s
$$

where $Z^{T}$ is the transpose of $Z$.

DEFINITION 2.7. Let $D(t, \phi)=D(t) \phi$ and consider the homogeneous difference equation

$$
\begin{aligned}
& D(t) x_{t}=0, \quad t>t_{0}, \\
& x_{t_{0}}=\phi, \quad D\left(t_{0}\right) \phi=0 .
\end{aligned}
$$

If $D(t) \phi$ is uniformly stable there are constants $\alpha, \beta$ such that for $t_{0} \in J=\left[t_{0}, t_{1}\right]$, $\phi \in C$, the solution of (2.10) satisfies $\left\|x_{t}\left(t_{0}, \phi\right)\right\| \leq \beta\|\phi\| e^{-\alpha\left(t-t_{0}\right)}, t \geq t_{0}$. 


\section{Controllability results}

LEMMA 3.1. System (2.3) is completely Euclidean controllable on $\left[t_{0}, t_{1}\right]$ if and only if $W$ is nonsingular.

PROOF. The proof can be observed from Proposition 3.1 of Dauer and Gahl [6].

LEMMA 3.2. System (2.3) is proper on $\left[t_{0}, t_{1}\right], t_{1}>t_{0}$, if and only if $0 \in$ int $R\left(t_{0}, t\right)$.

ProOF. Because $R\left(t_{0}, t\right)$ is a closed and convex subset of $E^{n}$, there exists a point $y_{1}$, on the boundary of $R\left(t_{0}, t\right)$, which implies that there is a support plane $\pi$ of $R\left(t_{0}, t\right)$ through $y_{1}$, that is, $\eta^{T}\left(y-y_{1}\right) \leq 0$ for each $y \in R\left(t_{0}, t\right)$, where $\eta \neq 0$ is an outward normal to $\pi$. If $u_{1}$ is the control corresponding to $y_{1}$ we have

$$
\eta^{T} \int Z(t, s) u(s) d s \leq \eta^{T} \int Z(t, s) u_{1}(s) d s
$$

for each $u \in C^{m}$. Since $C^{m}$ is a unit cube, this last inequality holds for each $u \in C^{m}$ if and only if

$$
\eta^{T} \int Z\left(t_{1}, s\right) u(s) d s \leq \int\left|\eta^{T} Z\left(t_{1}, s\right) u(s)\right| d s=y_{1}=\int\left|\eta^{T} Z\left(t_{1}, s\right)\right| d s
$$

and $u_{1}(s)=\operatorname{sgn} \eta^{T} Z\left(t_{1}, s\right)$.

Since we always have $0 \in R\left(t_{0}, t\right)$, if 0 were not in the interior of $R\left(t_{0}, t\right)$, then 0 would be on the boundary. Hence, from the preceding argument, this implies that $0=\int\left|\eta^{T} Z\left(t_{1}, s\right)\right| d s$, so that $\eta^{T} Z\left(t_{1}, s\right)=0$ a.e. $s \in\left[t_{1}, s\right]$. This by the definition of properness implies that the system is not proper. Since $\eta \neq 0$, this completes the proof.

THEOREM 3.3. System (2.3) is completely Euclidean controllable if and only if $0 \in$ int $R\left(t_{0}, t\right)$.

PROOF. Assume (2.3) is completely Euclidean controllable, then by Lemma 3.1, $W$ is nonsingular. We note that $W$ nonsingular is equivalent to $W$ being positive definite and this in turn is equivalent to $\eta^{T} Z\left(t_{1}, s\right)=0$ a.e. on $\left[t_{0}, t_{1}\right]$, which implies $\eta=0$. This, by definition, implies that system (2.3) is proper. Hence, by Lemma 3.2, this holds if and only if $0 \in \operatorname{int} R\left(t_{0}, t\right)$ and therefore (2.3) is completely Euclidean controllable with constraints.

THEOREM 3.4. In (2.3) assume that

(i) (2.3) is completely Euclidean controllable and 
(ii) (2.3) with $u=0$ is uniformly asymptotically stable.

Then (2.3) is Euclidean null controllable with constraints.

PROOF. By (i), system (2.3) is completely Euclidean controllable with constraints, so that $0 \in$ int $R\left(t, t_{0}\right), t \geq t_{0}$. Hence, there exists a ball $S$, such that $0 \in S \subseteq R\left(t, t_{0}\right)$, for each $t \geq t_{0}$. By (ii), every solution of (2.5) satisfies $x_{L}(t, 0) \rightarrow 0$ as $t \rightarrow \infty$. Hence at some $t_{1}<\infty, x_{L}\left(t_{1}, 0\right) \in S$. Therefore, using $t_{1}$ as an initial point and $x_{L_{t_{1}}}(\cdot, 0) \equiv \psi$ as an initial function, there exist a $u \in U$ and some $t_{2}>t_{1}$ such that the solution $x_{L}\left(t_{1}, x_{L_{t_{1}}}(\cdot, 0), u\right)$ of (2.4) satisfies $x_{L}\left(t_{2}, x_{L_{t_{1}}}(\cdot, 0), u\right)=0$, proving the theorem.

THEOREM 3.5. In system (2.3), if $\operatorname{rank}[F, G F]=n$, then (2.3) is completely Euclidean controllable on $\left[t_{0}, t_{1}\right]$.

PROOF. This is Theorem 2 of Gahl [9].

THEOREM 3.6. In system (2.3) assume that

(i) (2.3) with $u=0$ is uniformly asymptotically stable and

(ii) $\operatorname{rank}[F, G F]=n$.

Then (2.3) is Euclidean null controllable with constraints.

PROOF. By (ii), (2.3) is completely Euclidean controllable. Hence (i) and (ii) satisfy the requirements of Theorem 3.4 and the proof is complete.

COROLlaRY 3.7. For system (2.3) assume that

(i) the zero solution of (2.3) with $u=0$ is uniformly asymptotically stable,

(ii) $\operatorname{rank}[F, G F]=n$ and

(iii) $D(t, \phi)$ is uniformly stable.

Then system (2.3) is Euclidean null controllable with constraints.

PROOF. The proof follows immediately from Theorems 3.4 and 3.5.

COROLlaRY 3.8. Consider system (2.5), with all its assumptions. If there exists $v>0$ such that $|A(\theta)| \leq M \exp (v \theta) \leq M, \theta \in(-\infty, 0]$, and if

$$
B(\lambda)=\left\{\operatorname{Re} \lambda \geq 0, \operatorname{det}\left[\lambda\left(I-A e^{-\lambda}\right)-G-B e^{-\lambda}+\int_{-\infty}^{0} e^{\lambda \theta}[A(\theta) d \theta]=0\right]\right\}=\phi,
$$

then the solutions of (2.5) are uniformly asymptotically stable such that

$$
\left\|x_{t}\left(t_{0}, \phi\right)\right\| \leq k\|\phi\| e^{-\alpha(t-s)}, \quad t \geq t_{0},
$$

for some $\alpha>0, k>0$. 
PROOF. The proof can be observed from Sinha [14] and Onwuatu [13].

\section{Example}

Consider the neutral system

$$
\dot{x}(t)=L(x, u)+C_{0} \int_{-\infty}^{0} \exp (v \theta) x(t+\theta) d \theta,
$$

where $L(x, u)=A_{-1} \dot{x}(t-1)+A_{1} x(t-1)+A_{0} x(t)+B u(t)$ and

$$
\begin{aligned}
& A_{-1}=\left(\begin{array}{ll}
0 & 1 \\
1 & 0
\end{array}\right), \quad A_{0}=\left(\begin{array}{cc}
-1 & 1 \\
1 & -2
\end{array}\right), \quad A_{1}=\left(\begin{array}{cc}
0 & 3 \\
0 & -1
\end{array}\right), \\
& B=\left(\begin{array}{ll}
0 & 0 \\
1 & 0
\end{array}\right), \quad C_{0}=\left(\begin{array}{cc}
0 & 0 \\
0 & -1
\end{array}\right) .
\end{aligned}
$$

The characteristic root of the homogeneous equation

is

$$
\dot{x}(t)=A_{-1} \dot{x}(t-1)+A_{1} x(t-1)+A_{0} x(t)+\int_{-\infty}^{0} e^{v \theta} x(t+\theta) d \theta
$$

$$
\lambda^{2}+3 \lambda+1+\left(3 \lambda-\lambda^{2}\right) e^{-2 \lambda}+(2-3 \lambda) e^{-\lambda}+(\lambda+1) \int_{-\infty}^{0} e^{(\lambda+v) \theta} d \theta=0
$$

'Every root of (3.3) has a negative real part. Hence by Corollary 3.8, system (3.1) is uniformly asymptotically stable.

We now apply Theorem 3.5 to show Euclidean controllability of system (3.1) as follows. We require $\operatorname{rank}\left[B, A_{0} B\right]=n$. But

$$
\operatorname{rank}\left[B, A_{0} B\right]=\operatorname{rank}\left[\begin{array}{cccc}
0 & 0 & 1 & 0 \\
1 & 0 & -2 & 0
\end{array}\right]=\operatorname{rank}\left[\begin{array}{cccc}
0 & 0 & 1 & 0 \\
1 & 0 & 0 & 0
\end{array}\right]=2 .
$$

Since $\operatorname{rank}\left[B, A_{0} B\right]=n=2$, we conclude that system (3.1) by Theorem 3.6 is Euclidean null controllable with constraints.

\section{Conclusion}

We have developed and proved computable criteria for the Euclidean null controllability of infinite neutral systems with distributed delays. These conditions are given with respect to the stability of the free linear base system and the controllability of the linear controllable base system. 


\section{References}

[1] K. Balachandran and E. R. Anandhi, "Controllability of neutral functional integrodifferential infinite delay systems in Banach spaces", Taiwanese J. Math. 8 (2004) 689-702.

[2] T. A. Burton, Stability and periodic solutions of ordinary and functional differential equations (Academic Press, New York, 1985).

[3] E. N. Chukwu, "Control in $W_{2}^{(1)}$ of nonlinear interconnected systems of neutral type", J. Austral. Math. Soc. Series B 36 (1994) 286-312.

[4] E. N. Chukwu and H. C. Simpson, "Perturbations of nonlinear systems of neutral type", J. Differential Equations 82 (1989) 28-59.

[5] C. Corduneanu, Integral equations and applications (Cambridge University Press, Cambridge, 1991).

[6] J. P. Dauer and R. D. Gahl, "Controllability of nonlinear delay systems", J. Optim. Theory Appl. 21 (1977) 59-70.

[7] I. Davies and P. Jackreece, "Controllability and null controllability of linear systems", J. Appl. Sci. Environ. Mgt. 9 (2005) 31-36.

[8] X. Fu, "Controllability of neutral functional differential systems in abstract space", J. Appl. Math. Comput. 141 (2003) 281-296.

[9] R. D. Gahl, "Controllability of nonlinear system of neutral type", J. Math. Anal. Appl. 63 (1978) $33-42$.

[10] Y. Kuang and A. Feldstein, "Boundedness of solutions of nonlinear non-autonomous neutral delay equation", J. Math. Anal. Appl. 156 (1991) 293-304.

[11] V. Lakshmikantham, Theory of differential equations with unbounded delays (Kluwer Academic Publishers, Dordrecht, 1995).

[12] J. U. Onwuatu, "Null controllability of nonlinear infinite neutral system", Kybernetika 29 (1993) $325-336$.

[13] J. U. Onwuatu, "On criteria for closedness of an attainable set of a discrete neutral control system", J. Math. Anal. Appl. 268 (2002) 484-497.

[14] A. S. C. Sinha, "Null controllability of nonlinear infinite delay systems with restrained control", Int. J. Control 42 (1985) 735-741.

[15] J. Xu, Z. Wang and Z. Zheng, "On the existence of almost periodic solutions of neutral functional differential equations", EJQTDE 4 (1998) 1-9. 